\title{
Intralesional treatment with meglumine antimoniate in three patients with New World cutaneous leishmaniasis and large periarticular lesions with comorbidities
}

\author{
Maria Inês Fernandes Pimentel ${ }^{[1]}$, Érica de Camargo Ferreira e Vasconcellos ${ }^{[2]}$, \\ Carla de Oliveira Ribeiro ${ }^{[3]}$, Marcelo Rosandiski Lyra ${ }^{[1]}$, Mauricio Naoto Saheki ${ }^{[1]}$, \\ Mariza de Matos Salgueiro[1], Liliane de Fátima Antonio ${ }^{[1]}$ \\ and Armando de Oliveira Schubach ${ }^{[1]}$
}

[1]. Laboratório de Pesquisa Clínica e Vigilância em Leishmanioses, Instituto Nacional de Infectologia Evandro Chagas, Fundação Oswaldo Cruz, Rio de Janeiro, Rio de Janeiro, Brasil. [2]. Escola de Medicina Souza Marques, Rio de Janeiro, Rio de Janeiro, Brasil.

[3]. Centro de Medula Óssea, Instituto Nacional do Câncer, Rio de Janeiro, Rio de Janeiro, Brasil.

\begin{abstract}
Although New World cutaneous leishmaniasis is not itself a life-threatening disease, its treatment with systemic antimonials can cause toxicity that can be dangerous to some patients. Intralesional meglumine antimoniate provides a viable, less toxic alternative. Herein, we describe an alternative treatment with subcutaneous intralesional injections of meglumine antimoniate into large periarticular lesions of three patients with cutaneous leishmaniasis and comorbidities. This treatment was safe, successful, and well tolerated. This case series suggests that intralesional meglumine antimoniate is an effective therapy for cutaneous leishmaniasis, even with periarticular lesions. This hypothesis should be tested in controlled clinical trials.
\end{abstract}

Key words: Cutaneous leishmaniasis. Meglumine antimoniate. Intralesional injections.

\section{INTRODUCTION}

The first-choice treatment for American tegumentary leishmaniasis (ATL) is systemic pentavalent antimonials. However, these drugs are widely known for their toxic side effects. Recently, the World Health Organization recommended that decisions regarding treatment must be based mainly on the risk-benefit ratio of the intervention for each patient, and that local and less toxic treatments should be explored since mucocutaneous leishmaniasis occurs in less than $5 \%$ of cases, and systemic treatment does not prevent its occurrence ${ }^{1}$.

The National Institute of Infectious Diseases (INI), Oswaldo Cruz Foundation (Fiocruz), is a referral center for the treatment of ATL in Rio de Janeiro, Brazil. At this institution, intralesional (IL) treatment with meglumine antimoniate (MA) has been performed for over 30 years in selected patients with cutaneous leishmaniasis without mucosal lesions ${ }^{2}$, especially in those with contraindications to the systemic use of $\mathrm{MA}^{3}$. Recently, a standardized protocol of the IL therapy performed in the INI was published ${ }^{4}$.

Corresponding author: Dra. Maria Inês Fernandes Pimentel. e-mail: maria.pimentel@ini.fiocruz.br

Received 24 August 2016

Accepted 25 January 2017
The Pan-American Health Organization (PAHO) recommends that IL therapy should be administered via the intradermal route in referral centers for single lesions up to $900 \mathrm{~mm}^{2}$ in any location except the head and periarticular sites when immunosuppression is absent and patient follow-up is possible $^{5}$. We, however, describe three cases of parasitological confirmed cutaneous leishmaniasis and comorbidities, with larger periarticular lesions, successfully treated with IL MA via the subcutaneous route, and followed-up for at least 12 months after therapy.

\section{CASE REPORTS}

Three patients with cutaneous leishmaniasis were treated with IL MA (Aventis Pharma, São Paulo, Brazil), supplied by the Brazilian Ministry of Health, via the subcutaneous route according to standard techniques that have been previously reported $^{4}$. All patients signed informed consent forms for diagnostic procedures and treatment. There was no need for local anesthesia in any patient, and the procedure had good acceptability. No patient relapsed in the subsequent 12-month follow-up. The characteristics of the patients before and after IL treatment with MA are shown in Table 1. The aspects of cutaneous lesions before and after treatment (at the time of complete healing) are shown in Figure 1. 


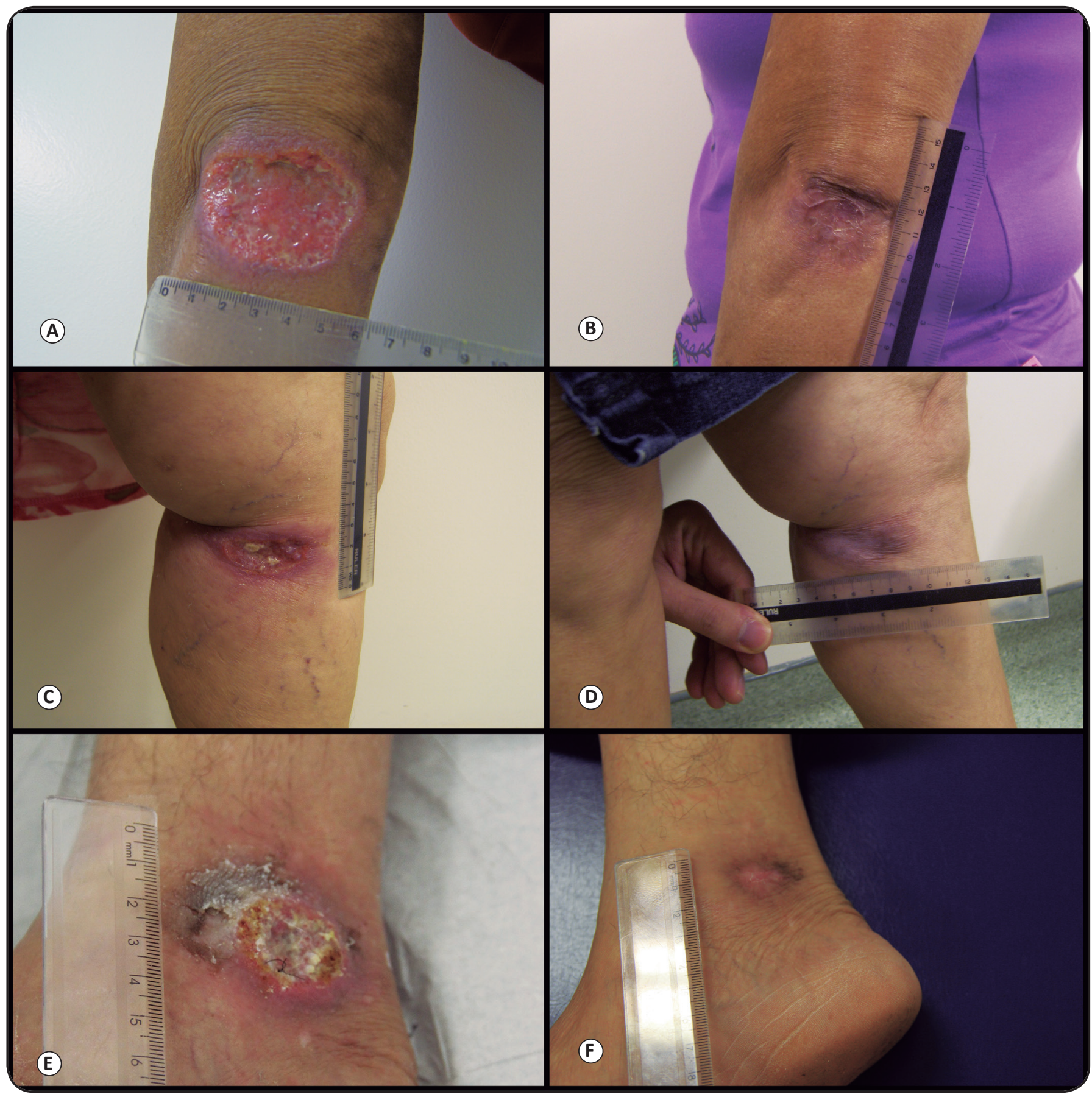

FIGURE 1 - Cutaneous leishmaniasis with large periarticular lesions in three patients treated with intralesional meglumine antimoniate, before and after therapy. Figure 1A, C, and E: Patients 1, 2, and 3, respectively, pre-treatment. Figure 1B, D, and F: Patients 1, 2, and 3, respectively, post-treatment (complete healing).

\section{DISCUSSION}

Herein, we describe three cases of parasitological confirmed cutaneous leishmaniasis with large periarticular lesions that were successfully treated with IL MA despite the presence of comorbidities and the paucity of treatment options. All patients were followed-up for at least one year after treatment without relapse or development of mucosal lesions. In the INI/Fiocruz, ear, nose, and throat specialists routinely perform a fiber optic examination of the upper aero-digestive pathways once a year for at least five years after clinical cure. In our institution, the evolution of treated cutaneous leishmaniasis to mucosal leishmaniasis is a very rare event.

Because of the virtual absence of mucosal leishmaniasis in the Old World, cutaneous leishmaniasis can frequently be managed with local wound care or topical specific treatment ${ }^{1}$. In the New World, the rate of occurrence of mucosal leishmaniasis 


\section{TABLE 1}

Characteristics of three patients with New World cutaneous leishmaniasis with large periarticular lesions treated with intralesional meglumine antimoniate.

\begin{tabular}{|c|c|c|c|}
\hline Characteristics & Patient 1 & Patient 2 & Patient 3 \\
\hline Gender & female & female & male \\
\hline Area of the lesion* & $3,023.8 \mathrm{~mm}^{2}$ & $1,178.1 \mathrm{~mm}^{2}$ & $942.5 \mathrm{~mm}^{2}$ \\
\hline Location of the lesion & Extensor surface of the left elbow & $\begin{array}{l}\text { Upper third, medial face, } \\
\text { left leg, near the knee }\end{array}$ & Right medial malleolus \\
\hline Method of parasitological confirmation & $\begin{array}{l}\text { Culture for Leishmania genus } \\
\text { positive in NNN }\end{array}$ & $\begin{array}{l}\text { Culture for Leishmania } \\
\text { genus positive in NNN }\end{array}$ & $\begin{array}{l}\text { Presence of amastigotes on } \\
\text { histopathology }\end{array}$ \\
\hline Time interval between infiltrations (days) & 21 & 21 & 21 \\
\hline Time to epithelialization (months after treatment) & 3 & 1 & 2.5 \\
\hline Time to complete healing (months after treatment) & 8 & 3 & 3.5 \\
\hline Follow-up (months after treatment) & $12^{* *}$ & 66 & 12 \\
\hline
\end{tabular}

IL: intralesional; MA: meglumine antimoniate; NNN: Novy, McNeal, and Nicolle medium. *Area of the ellipse - formula: major axis/2 X minor axis/2 X $\pi$. ** Patient 1 died because of previous comorbidities after 12 months of follow-up.

limits the option of not providing specific treatment to a patient with cutaneous leishmaniasis.

Cutaneous leishmaniasis in Rio de Janeiro is almost exclusively due to Leishmania braziliensis $^{6}$. A recent systematic review of American cutaneous leishmaniasis revealed a very low $(6 \%)$ spontaneous cure rate for $L$. braziliensis infection? ${ }^{7}$. Specific treatment is thus almost mandatory. Patients with contraindications to systemic treatment, however, can benefit from IL MA treatment ${ }^{3,8}$. Recently, Soto et al. ${ }^{9}$ found that treatment with IL MA has comparable efficacy to treatment with IL pentamidine in Bolivian patients with cutaneous leishmaniasis caused by L. braziliensis.

The PAHO recommendations ${ }^{5}$ were based on the scarce literature available up to 2013 on IL treatment of ATL. However, PAHO also invited researchers to report local experiences in order to establish local and regional profiles. Our experience differs from the PAHO recommendations for IL MA therapy on the following issues: 1) contraindication for periarticular lesions; 2) contraindication for lesions $>900 \mathrm{~mm}^{2}$; and 3) the use of the intradermal route. Notably, the periarticular location of the lesions did not prevent epithelialization or complete healing in our patients. The large area of the lesions in patients 2 and 3 did not hinder healing process either, although the lesion in patient 1 , which was larger than the lesions in the other two patients, took longer to heal. Finally, intradermal infiltration of a considerable amount of medication $(>1.0 \mathrm{~mL})$ would not be advisable, if at all feasible. We hypothesize that passive diffusion from the hypodermis could explain the success of IL therapy via the subcutaneous route, which is much easier to perform than intradermal injection is. In addition, although IL therapy can be painful, the discomfort caused by the subcutaneous injection was well tolerated, and local anesthesia was not needed in any patient.

We therefore recommend IL MA when comorbidities hamper or contraindicate systemic treatment, even in larger periarticular lesions. The results obtained with the treatment of these three patients suggest that IL treatment with MA in periarticular lesions, when systemic treatment is not feasible, may be successful, safe, and well tolerated. Good monitoring of the skin lesions and inquiry into complaints of the mucous membranes in the upper aero-digestive tract is desirable for at least one year after treatment. In addition, patients should be instructed to seek medical advice whenever they experience nose- and throat-related symptoms. IL use of MA in ATL should be better studied through controlled clinical trials, including in patients with periarticular lesions.

\section{Acknowledgments}

The authors would like to thank Cláudia Maria Valete-Rosalino for the ear, nose, and throat evaluation of all patients; and the staff members of Laboratório de Pesquisa Clínica e Vigilância em Leishmanioses, Instituto Nacional de Infectologia Evandro Chagas, Fundação Oswaldo Cruz for laboratory and administrative support. 


\section{Financial support}

This study was supported by Instituto Nacional de Infectologia Evandro Chagas, FIOCRUZ.

\section{Conflicts of interest}

The authors declare that there is no conflict of interest.

\section{REFERENCES}

1. World Health Organization (who). Control of the leishmaniases. WHO Technical Report Series 949. Geneva: 2010. p. 49-90.

2. Oliveira-Neto MP, Schubach A, Mattos M, Gonçalves da Costa SC, Pirmez C. Intralesional therapy of American cutaneous leishmaniasis with pentavalent antimony in Rio de Janeiro, Brazil an area of Leishmania (V.) braziliensis transmission. Int J Dermatol. 1997;36(6):463-8.

3. Vasconcellos ECF, Pimentel MIF, Schubach AO, Oliveira RVC, Azeredo-Coutinho RB, Conceição-Silva $\mathrm{F}$, et al. Intralesional meglumine antimoniate for treatment of cutaneous leishmaniasis patients with contraindication to systemic treatment from Rio de Janeiro (2000-2006). Am J Trop Med Hyg. 2012;87(2):257-60.
4. Duque MC, Vasconcellos ECF, Pimentel MIF, Lyra MR, Pacheco SJB, Marzochi MCA, et al. Standardization of intralesional meglumine antimoniate treatment of cutaneous leishmaniasis. Rev Soc Bras Med Trop. 2016;49(6):774-6.

5. Organización Pan-Americana de la Salud. Leishmaniasis en las Américas: recomendaciones para el tratamento. Washington DC: 2013. p. 6-15.

6. de Oliveira-Neto MP, Mattos MS, Da-Cruz AM, Fernandes O, Moreira J, Gonçalves-Costa SC, et al. American tegumentar leishmaniasis (ATL) in Rio de Janeiro State, Brazil: main clinical and epidemiologic characteristics. Int J Dermatol. 2000;39(7):506-14.

7. Cota GF, de Sousa MR, Fereguetti TO, Saleme PS, Alvarisa TK, Rabello A. The cure rate after placebo or no therapy in American cutaneous leishmaniasis: a systematic review and meta-analysis. PLOS One. 2016;11(2):e0149697.

8. Silva RE, Toledo Jr A, Senna MC, Rabello A, Cota G. Intralesional meglumine antimoniate for the treatment of localised cutaneous leishmaniasis: a retrospective review of a Brazilian referral centre. Mem Inst Oswaldo Cruz. 2016; 111(8):512-6

9. Soto J, Paz D, Rivero D, Soto P, Quispe J, Toledo J, et al. Intralesional pentamidine: A novel therapy for single lesions of Bolivian cutaneous leishmaniasis. Am J Trop Med Hyg. 2016;94(4):852-6. 